\title{
The pigment epithelial-derived factor gene loaded in PLGA nanoparticles for therapy of colon carcinoma
}

\author{
FENG-YU CUI ${ }^{2 *}$, XIANG-RONG SONG ${ }^{*}$, ZHI-YONG LI ${ }^{1}$, SHUANG-ZHI LI ${ }^{1}$, \\ BO MU², YONG-QIU MAO ${ }^{1}$, YU-QUAN WEI ${ }^{1}$ and LI YANG $^{1}$ \\ ${ }^{1}$ State Key Laboratory of Biotherapy, West China Hospital, and \\ ${ }^{2}$ School of Life Science, Sichuan University, Chengdu, P.R. China
}

Received February 8, 2010; Accepted March 19, 2010

DOI: $10.3892 /$ or_00000905

\begin{abstract}
Colon carcinoma is one of the common malignant tumors and has high morbidity and mortality in the world. Pigment epithelial-derived factor (PEDF) has been found to be the most potent natural inhibitor of angiogenesis and PEDF gene has been extensively used for the therapy of tumors, which suggests a potential approach to the therapy of colon carcinoma. However, the transfer of PEDF gene largely depends on the effective gene delivery systems. Poly (lactic-co-glycolic acid) nanoparticles (PLGANPs) have been extensively used for gene therapy due to its lowtoxicity, biocompatibility and biodegradability, due to its potential to be an excellent carrier of the PEDF gene. We investigated the effect of PEDF gene loaded in PLGA nanoparticles (PEDF-PLGANPs) on the mouse colon carcinoma cells (CT26s) in vitro and in vivo. Blank PLGANPs (bPLGANPs) showed lower cytotoxicity than PEI to the CT26s. In vitro, PEDF-PLGANPs directly induced CT26 apoptosis and inhibit human umbilical vein endothelial cell (HUVEC) proliferation. In vivo, PEDFPLGANPs inhibited CT26 tumors growth by inducing CT26 apoptosis, decreasing MVD and inhibiting angiogenesis. Our present study demonstrates the inhibitory effect of PEDF-PLGANPs on the growth of CT26s in vitro and in vivo
\end{abstract}

Correspondence to: Professor Li Yang, State Key Laboratory of Biotherapy, West China Hospital, Sichuan University, Keyuan Road 4, Chengdu, Sichuan 610041, P.R. China

E-mail: yl_tracy@hotmail.com

${ }^{*}$ Contributed equally

Abbreviations: PLGA, poly (lactic-co-glycolic acid); PEDF, pigment epithelial-derived factor; PLGANPs, PLGA nanoparticles; bPLGANPs, blank PLGA nanoparticles; PEDF-PLGANPs, PEDF gene loaded in PLGA nanoparticles; $\mathrm{AAV}_{2}$-PLGANP s, $\mathrm{AAV}_{2}$ gene loaded in PLGA nanoparticles; NS, normal saline; CT26s, mouse colon carcinoma cells; HUVECs, human umbilical vein endothelial cells; MVD, microvessels density

Key words: poly (lactic-co-glycolic acid), PLGA nanoparticles, pigment epithelial-derived factor, CT26s, tumor, apoptosis, angiogenesis for the first time. PLGANP-mediated PEDF gene could provide an innovative strategy for the therapy of colon carcinoma.

\section{Introduction}

Colon carcinoma is one of the common malignant tumors and has high morbidity and mortality world-wide. The main treatment of colon carcinoma is surgical resection of the primary tumor. However, it is often unsatisfactory because of the risk of high relapse or the development of metastases after attempted curative resections. Gene therapy possibly targeted at the level of cellular gene expression suggests a new approach against tumors (1), which could provide a novel way for the treatment of colon carcinoma.

Pigment epithelial-derived factor (PEDF) has been reported to be the most potent natural anti-angiogenic agent far greater than any other known endogenously produced factor $(2,3)$ and induce tumor cell apoptosis $(4,5)$, which efficiently inhibited the growth of tumors. It has been reported that PEDF gene had better therapeutic efficacy on many tumors such as pancreas and prostate (6), melanoma $(4,5)$ and hepatocellular carcinoma (7). However, the effect of PEDF gene on colon carcinoma has not been investigated. We hypothesized that PEDF gene could also play a therapeutic effect on colon carcinoma by the above mechanisms.

The transfer of PEDF gene is involved in viral vector $(8,9)$ and non-viral vector $(10,11)$. Some concerns associated with viral vectors such as potential oncogenicity and toxicity (12) have encouraged researchers to increasingly focus on plasmid vectors for safety. However, the benefits of plasmid DNA (pDNA) as drugs will largely depend on an effective delivery system to enhance its ability to the cells, including PEDF gene. Polyethylenimine (PEI) has been extensively studied in vitro and in vivo as the carriers of pDNA $(13,14)$. Nevertheless, the non-biodegradability of PEI polymers results in fairly high toxicity in vivo $(15,16)$, which has become the hindrance of it for further application in vivo. It has become one of the goals to develop a gene delivery system that safely and reliably express of the transgene.

Nanoparticles made from poly (d, 1-lactide-co-glycolide acid) (PLGANPs) have been the focus of extensive researches in gene delivery and expression in vitro and in vivo (17-19) 
due to its excellent low-toxicity, biodegradability and biocompatibility. Moreover, PLGANPs could rapidly escape the endo-lysosomal pathway and delivers different classes of therapeutic agents into the cytoplasm and nucleus (20), which could enhance the expression and utilization of pDNA as drugs. However, it is not investigated whether PLGANPmediated PEDF gene could achieve satisfactory inhibitory effect on tumors. Therefore, PLGANPs encapsulating PEDF gene were used for the treatment of colon carcinoma for the first time in our study.

In this study, taking account of the above agents, we investigated whether PEDF gene loaded in PLGANPs can play an inhibiting role on mouse colon carcinoma cells (CT26s) in vitro and in vivo. PLGANP-mediated PEDF gene provides an innovative strategy for the therapy of colon carcinoma.

\section{Materials and methods}

Cell culture. CT26s and HUVECs were purchased from American Type Culture Collection (ATCC, Rockville MD, USA) and preserved in our laboratory. They were respectively cultured in RPMI-1640 and DMEM medium (GibcoBRL, Grand Island, NY, USA) supplemented with $10 \%$ (v/v) fetal bovine serum (FBS) plus $100 \mu \mathrm{g} / \mathrm{ml}$ amikacin. The cells were maintained in the $37^{\circ} \mathrm{C}$ humidified incubator with $5 \% \mathrm{CO}_{2}$ atmosphere.

pDNA construction. Human PEDF cDNA was PCR-amplified and then inserted into the expression plasmid vector $\mathrm{pAAV}_{2}$ $\left(\mathrm{pAAV}_{2}-\mathrm{PEDF}\right)$. The pDNA $\left(\mathrm{pAAV}_{2}\right.$ and $\left.\mathrm{pAAV}_{2}-\mathrm{PEDF}\right)$ were considerably propagated in E. coli $\mathrm{DH} 5 \alpha$ and purified using Endofree Plasmid Giga kit (Qiagen, Chatsworth, CA). The purity and concentration were confirmed by measuring absorbance at 260 and $280 \mathrm{~nm}$ on Ultrospec 3100 pro (Amersham Biosciences, USA) and agarose gel electrophoresis.

pDNA-PLGANP preparation. The PLGA nanoparticles encapsulating pDNA (pDNA-PLGANPs) were prepared by a modified version of a w/o/w double-emulsion solvent evaporation process (21). The pDNA was incubated with equivalent poly-L-lysine (PLL; MW 25,000, Sigma) to be condensed, which formed the inner aqueous phase. The compound was added dropwise into chloroform-acetone mixture (9:1, v/v) containing $10 \mathrm{mg}$ PLGA (LA: GA=75:25, MW 15,000 Da, Department of Medical Polymers Shan Dong Institute, China) and was emulsified by probe sonication. The resulting primary emulsion was mixed with polyvinyl alcohol (PVA, Sigma, USA) dissolved in TE buffer (PVATE) and further emulsified by probe sonication, which formed $\mathrm{W} / \mathrm{O} / \mathrm{W}$ double emulsion. Then, PVA-TE was added into $\mathrm{W} / \mathrm{O} / \mathrm{W}$ double emulsion and continued to be emulsified by probe sonication. The probe sonication was performed in an ice bath. The suspension was finally stirred to remove the organic solvent.

pDNA-PLGANP characterizations. The entrapment efficiencies of pDNA could be calculated with the percent ratio of the actual amounts of pDNA incorporated into nanoparticles to the total amounts of pDNA in nanoparticle suspension (22). The nanoparticle supernatant was quantitated using Hoechst 33258 staining solution (Sigma) after ultracentrifugation (Optima $^{\mathrm{TM}}$ L-80 XP Ultracentrifuge, Beckman Coulter, USA) and examined by absorbance at $260 \mathrm{~nm}$ using UV-spectroscopy (RF-5301PC, Japan) to determine the amount of pDNA unencapsulated. The appearance of the nanoparticles was observed by scanning electron micrograph (SEM; S-4800, Hitachi, Japan). The mean diameter of pDNA-PLGANPs was evaluated by photon correlation spectroscopy (PCS) and a potential analyzer (Zetasizer Nano ZS90, Malvern Instruments Ltd., Malvern, UK). Then, pDNA was extracted from PLGANPs as reported previously (23). In brief, centrifuged PLGANPs were dissolved in $0.5 \mathrm{~N} \mathrm{NaOH}$ under stirring at $37^{\circ} \mathrm{C}$ until a clear solution was obtained. pDNA from PLGANPs was analyzed by $1.0 \%$ $(\mathrm{w} / \mathrm{v})$ agarose gel electrophoresis and observed by a gel documentation system (Gel Doc 1000, Bio-Rad Laboratories, Hercules, CA, USA) to determine its integrity and purity. Experiments were performed in triplicate.

bPLGANP cytotoxicity. The cytotoxicity of bPLGANPs was evaluated by MTT assay. In brief, the CT26s were seeded into 96-well plates and incubated for $24 \mathrm{~h}$. Then, the cells were exposed to bPLGANPs and PEI (Mw 25,000 Da, Sigma) (expressed as PLGA and PEI concentrations at 0.5 , 2 and $10 \mathrm{mg} / \mathrm{ml}$ ) for another $48 \mathrm{~h}$. The CT26s without any treatment and only be cultured with complete medium were the control. The relative cell viability compared to control was calculated as $\mathrm{A}_{\text {sample }} / \mathrm{A}_{\text {control }} \times 100 \%$. All the experiments were repeated in triplicates.

Flow cytometry analysis. CT26s in 6-well plates were transfected with PEDF-PLGANPs and $\mathrm{AAV}_{2}$-PLGANPs. The CT26s treated with normal saline were the control. After another $48 \mathrm{~h}$, the cells were washed with PBS for three times and resuspended in PI buffer $(50 \mathrm{mg} / \mathrm{ml} \mathrm{PI,} 0.1 \%$ Triton X-100, $0.1 \mathrm{mM}$ EDTA $(\mathrm{Na})_{2}$ and $50 \mathrm{mg} / \mathrm{ml} \mathrm{RNase} \mathrm{A)} \mathrm{for} 30 \mathrm{~min}$ at $4^{\circ} \mathrm{C}$ protected from light until the analysis by flow cytometry (24).

HUVEC proliferation assay. HUVECs were used to evaluate the effect of PEDF-PLGANPs on angiogenesis in vitro by MTT assay. Briefly, HUVECs were seeded into 96-well plates for $24 \mathrm{~h}$. PEDF-PLGANPs, AAV $2^{-}$ PLGANPs and NS diluted into a series of $1 / 2$ concentrations from 1:2 to $1: 64$ were added into each well. The cells were incubated for another $48 \mathrm{~h}$. After adding MTT to each well, the cells were incubated at $37^{\circ} \mathrm{C}$ for $3 \mathrm{~h}$ and then treated as described in bPLGANPs cytotoxicity. All the experiments were repeated in triplicates.

Tumor studies in vivo. Six to eight week-old BALB/c mice were purchased from the West China Experimental Animal Center of Sichuan University (Sichuan, China). All animal procedures were conducted according to guidelines provided by the West China Hospital Cancer Center's Animal Care and Use Committee. All measures were taken to minimize any pain or discomfort. CT26s was injected into the right flank of each mouse. The mice were then randomly divided into three groups: $\mathrm{NS}$; $\mathrm{AAV}_{2}$-PLGANPs; PEDF-PLGANPs when the tumors reached an average diameter of $3 \mathrm{~mm}$. Then the tumors were treated by intratumoral injection 

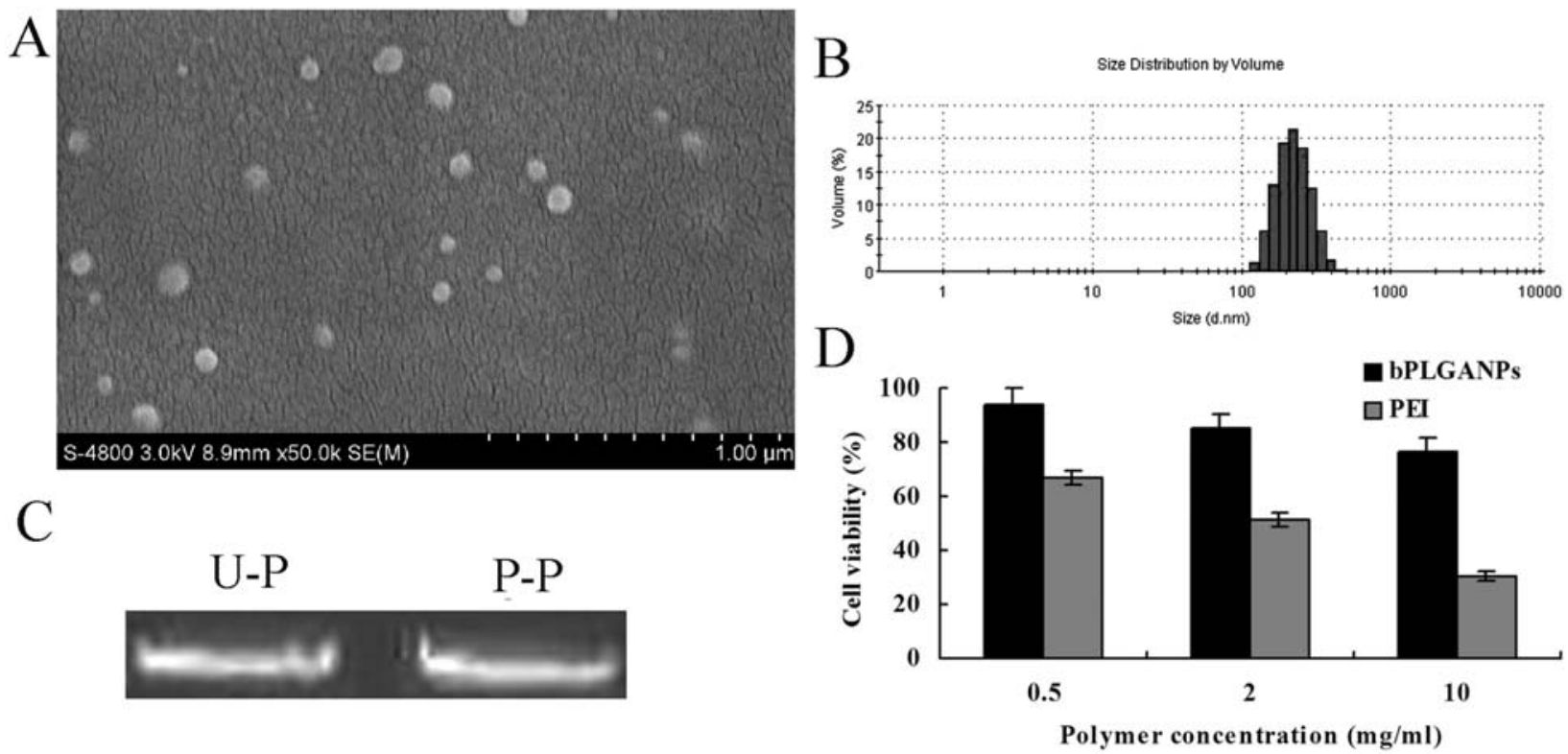

Figure 1. The characterizations of PLGANPs. (A) Scanning electron micrograph (SEM) of pDNA-PLGANPs. (B) Size distribution of PLGANPs by volume (\%). (C) Agarose gel electrophoresis was used to evaluate the integrity and purity of $\mathrm{pAAV}_{2}-\mathrm{PEDF}$ extracted from PLGANPs. They were pAAV $\mathrm{P}_{2} \mathrm{PEDF}$ extracted from PLGANPs (P-P) and untreated $\mathrm{pAAV}_{2}-\mathrm{PEDF}$ (U-P), respectively. (D) MTT assay was used to examine the cell cytotoxicity of bPLGANPs and PEI at different concentrations against CT26s, $48 \mathrm{~h}$ post-treatment $(\mathrm{n}=3)$. The data are expressed as mean \pm SD.

every two days. Tumors sizes were measured every three days and the volume was calculated as $0.52 \mathrm{x}$ length $\mathrm{x}$ width ${ }^{2}(25)$. Subcutaneous tumor tissues were weighed and then fixed in $4 \%$ paraformaldehyde for further study when the mice were sacrificed.

Western blotting analysis. Western blotting was used to confirm the successful expression of PEDF gene loaded in PLGANPs in vitro and in vivo. The CT26s of PEDFPLGANPs, $\mathrm{AAV}_{2}$-PLGANPs and NS groups were lysed with RIPA solution (150 mmol/1 NaCl; $1.0 \% \mathrm{NP}-40 ; 0.5 \%$ NaTDC; $0.1 \%$ SDS; 50 mmol/1 Tris, $\mathrm{pH} \mathrm{8.0)} \mathrm{while} \mathrm{the} \mathrm{CT26} \mathrm{tumors}$ of PEDF-PLGANPs, $\mathrm{AAV}_{2}$-PLGANPs and NS groups were respectively grinded and then lysed with RIPA solution. Supernatants were respectively collected after centrifugalization and subjected to Western blotting analysis. The proteins were separated by SDS-polyacrylamide gel electrophoresis (SDS-PAGE) and transferred to a polyvinylidene difluoride membrane (PVDF, Bio-Rad, Richmond, CA, USA). Expressed PEDF protein was probed with a primary monoclonal antibody mouse against human PEDF (3:1000, mAb; R\&D Systems, Boston, MA, USA) and a peroxidaseconjugated secondary antibody goat anti-mouse IgG (1:10,000, ZSGB-BIO, Beijing, China). The bands were observed using an enhanced chemiluminescence (ECL) detection system (Pierce, Rockford, IL, USA).

Histology and CD31 immunochemistry staining. Tumors fixed in $4 \%$ paraformaldehyde were embedded in paraffin, cut into $3-5-\mu \mathrm{m}$ sections and subjected to hematoxylin and eosin (H\&E) and CD31 immunohistochemical staining as described previously (26). In brief, the slides of CT26 tumors were incubated with goat anti-mouse CD31 monoclonal antibody (1:100, Boster Biological Engineering Co., Wuhan, China) at $4{ }^{\circ} \mathrm{C}$ overnight. Biotinylated polyclonal rabbit anti- goat (1:100, Boster Biological Engineering Co.) and ABC kit (1:100, Boster Biological Engineering Co.) were respectively utilized to incubate the sections of tumors. The microvessels were observed by DAB visualization system (ZSJQ Biotechnology, Beijing, China). The stained micro-vessels were counted in high-power (x400) fields and CD31-stained endothelial cells or endothelial cell clusters were considered a single countable microvessel. The microvessel density (MVD) was expressed as the number of microvessels per field according to the method of Weidner et al (27).

Apoptosis assay. To detect the apoptotic cells in the subcutaneous tumors, paraffin sections were processed using the DeadEnd Colorimetric Terminal Deoxynucleotidyl Transferase-Mediated dUTP Nick-End Labeling (TUNEL) System (Promega, Corp., Madison, WI, USA). The number of apoptotic cells in ten high power fields of each slide and three slides of each animal were examined. The apoptosis index was calculated with the percent ratio of the number of apoptotic cells to the total number of cells in the field (4).

Statistical analysis. SPSS software (SPSS Inc., Chicago, IL, USA) was used for statistical analysis. Quantitative data are expressed as mean \pm standard deviation (SD). One-way analysis of variance (ANOVA) was performed to determine statistical significances. A value of $p<0.05$ was considered statistically significant.

\section{Results}

Nanoparticle characterization. The morphological characterization of pDNA-PLGANPs were observed by SEM, which showed the shape and smooth surface and steadily dispersed suspension (Fig. 1A). PCS exhibited that pDNAPLGANPs had an average diameter of $220.95 \pm 7.20 \mathrm{~nm}$ and 

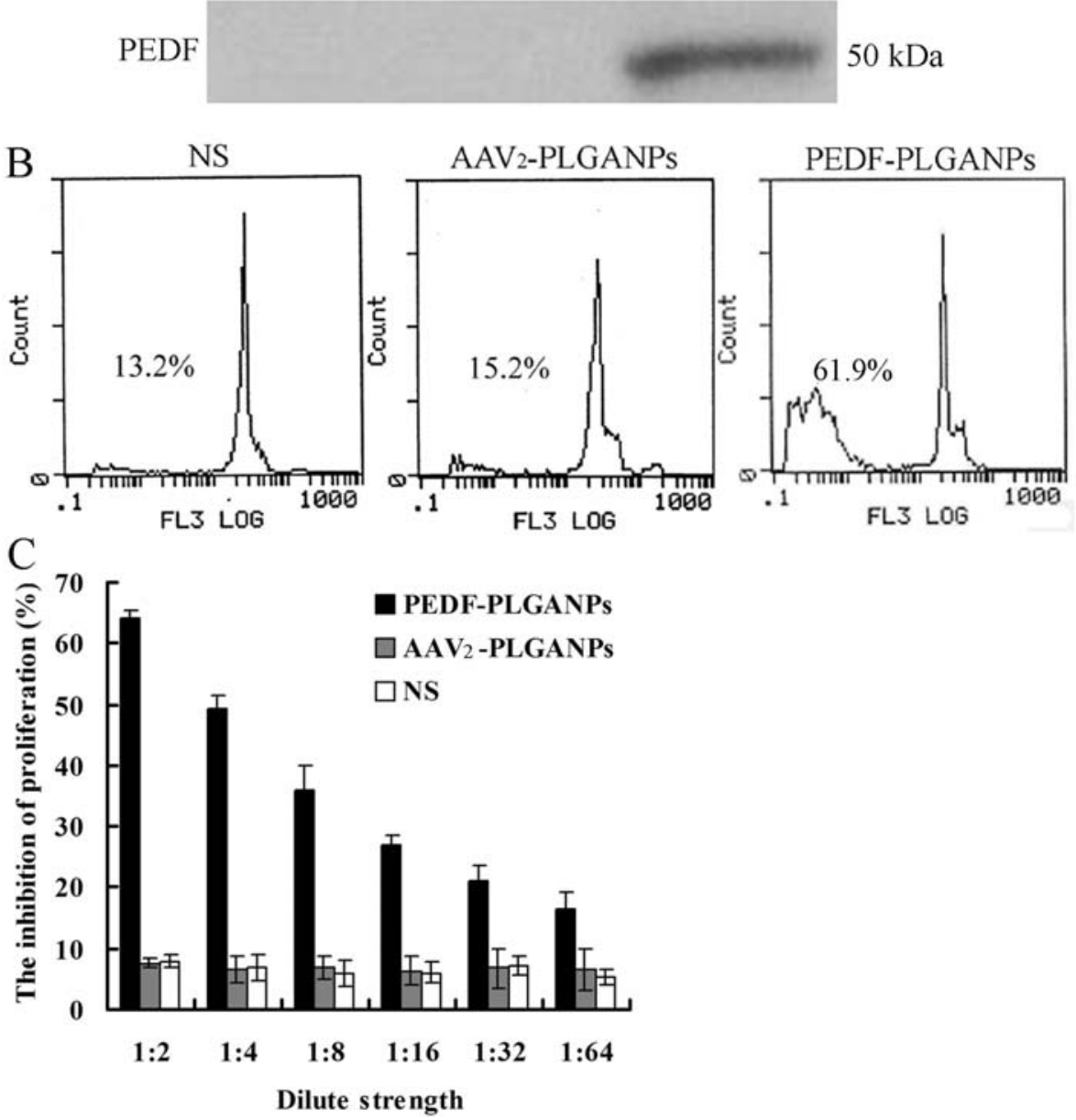

Figure 2. The effects of PEDF-PLGANPs in vitro. (A) The supernatants from CT26s of NS, AAV ${ }_{2}$-PLGANPs and PEDF-PLGANPs group were, respectively, detected by SDS-PAGE. PEDF protein $(50 \mathrm{kDa})$ was only probed in CT26s treated with PEDF-PLGANPs. (B) Apoptotic CT26s of PEDF-PLGANPs, $\mathrm{AAV}_{2}$-PLGANPs and NS group were evaluated by flow cytometry with the values of $61.9,15.2$ and $13.2 \%$, respectively. (C) The inhibitory effect of PEDF-PLGANPs on the proliferation of HUVECs was performed with a series of 1/2 dilutions from 1:2 to 1:64. The inhibitory rates of PEDF-PLGANP treated group declined with the increase of dilutions while $\mathrm{AAV}_{2}$-PLGANPs or NS group had no significantly change.

the polydispersity index (PDI) of $0.14 \pm 0.03$ represented a narrow size distribution (Fig. 1B). pDNA was encapsulated into PLGANPs with a high entrapment efficiency of 88.03\% \pm 2.82 . As shown in Fig. 1C, $\mathrm{pAAV}_{2}-\mathrm{PEDF}$ extracted from PLGANPs had a single band and was similar to the $\mathrm{pAAV}_{2}-\mathrm{PEDF}$ unencapsulated in PLGANPs, which revealed excellent structural integrity and purity. The cell cytotoxicity of bPLGANPs and PEI at different concentrations against CT26s is shown in Fig. 1D. According to Fig. 1D, the CT26s treated with bPLGANPs showed higher cell viability rate than the cells treated with PEI at the same concentration and the CT26 viability decreased slightly with the increased concentration of bPLGANPs, which manifested that PLGANPs were relatively safer than PEI as the delivery system to the CT26s.

PEDF gene loaded in PLGANPs could be successfully expressed, inducing CT26 apoptosis and inhibiting HUVEC proliferation in vitro. The expression of PEDF gene loaded in PLGANPs was detected by SDS-PAGE. As shown in Fig. 2A, PEDF protein was detected in CT26s of PEDFPLGANPs group, but not in $\mathrm{AAV}_{2}$-PLGANPs or NS group.
The result suggested that PEDF-PLGANPs could be internalized into the CT26s and PEDF protein was successfully expressed.

Flow cytometry analysis was used to evaluate whether PEDF gene could induce CT26 apoptosis and carry out the quantitative assessment of apoptotic CT26s in vitro. As shown in Fig. 2B, CT26s transfected with PEDF-PLGANPs have a conspicuous peak $(61.9 \%)$ compared with $\mathrm{AAV}_{2}$-PLGANPs or NS groups, with the values of 15.2 and $13.2 \%$, respectively.

To evaluate the influence of PEDF gene in PLGANPs on angiogenesis in vitro, proliferation of HUVECs exposed to the nanoparticles were assessed by MTT assay. As shown in Fig. 2C, HUVECs exposed to PEDF-PLGANPs had a significant inhibition effect with an inhibitory rate of $64.2 \pm 1.5 \%$ at a $1: 2$ dilution. In addition, the inhibitory rates declined with the increase of dilutions, suggesting a dosedependent effect. Differently, the inhibition rate of $\mathrm{AAV}_{2^{-}}$ PLGANPs or NS group had no significantly change with the series of $1 / 2$ concentrations from $1: 2$ to $1: 64$. The results indicated that PEDF-PLGANPs could play an inhibitory role on the proliferation of HUVECs, which possibly suggested an inhibited effect on angiogenesis within tumors. 
A
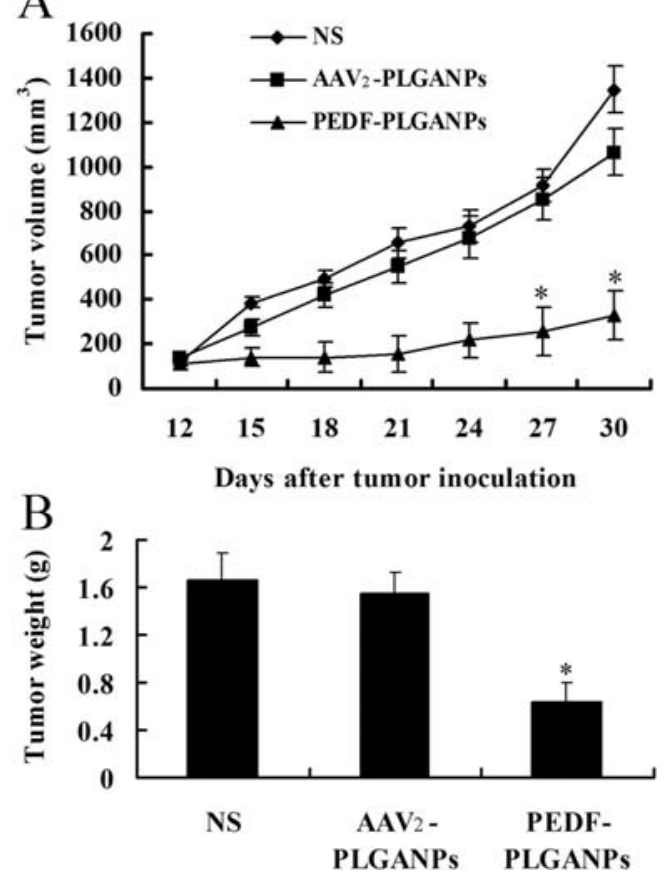

Figure 3. The study of PEDF-PLGANPs on the mice bearing CT26 tumors. (A) Tumor volumes of the mice bearing CT26 tumors in each group. (B) The tumors of experimental mice were weighed on the day 33 after CT26 inoculation when the mice were sacrificed. The values are expressed as mean $\pm \mathrm{SD} .{ }^{*} \mathrm{P}<0.05$ compared with the tumor weight of $\mathrm{AAV}_{2}-\mathrm{PLGANPs}$ or NS group.

The inhibition effect of PEDF-PLGANPs on primary CT26 tumors growth in vivo. To further examine the therapeutic effect of PEDF gene, PEDF-PLGANPs were injected into BABL/C mice bearing CT26 subcutaneous tumors. During the experiment, the growth condition of the mice was good and PEDF-PLGANPs showed enhanced suppressed efficacy on tumor volumes (Fig. 3A). Compared with NS or AAV2PLGANPs treated mice, the tumor volumes of PEDFPLGANPs treated mice increased slightly all the time. The tumor volume of PEDF-PLGANPs treated mice reached $500 \mathrm{~mm}^{3}$ vs. nearly $2000 \mathrm{~mm}^{3}$ in the other two groups on day 33 after CT26 inoculation, which represents a significant difference $(\mathrm{p}<0.05)$. Furthermore, the mean tumor weight of PEDF-PLGANPs treated mice was lighter than that of $\mathrm{NS}$ or $\mathrm{AAV}_{2}-\mathrm{PLGANPs}$ treated mice $(\mathrm{p}<0.05)$ as shown in Fig. 3B.

PEDF gene in PLGANPs could be successfully expressed in vivo. SDS-PAGE was performed to confirm the expression of PEDF gene in vivo. PEDF protein was only probed in CT26 tumors from PEDF-PLGANP-treated mice (Fig. 4). The result indicated that PEDF gene was successfully expressed in vivo.

The growth inhibition of CT26 tumors was related to CT26s apoptosis, decreasing MVD and angiogenesis inhibition caused by PEDF-PLGANPs in vivo. TUNEL assay was used to evaluate whether the growth inhibitory effect of CT26 tumors was associated with the apoptosis of CT26s in vivo. The number of apoptotic cells within the tumors of PEDF-

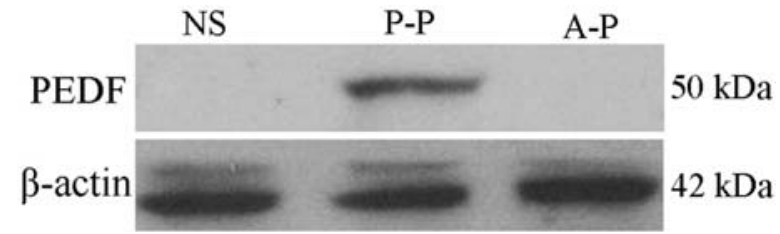

Figure 4. The expression of PEDF gene loaded in PLGANPs in vivo. PEDF protein $(50 \mathrm{kDa})$ could be only detected in CT26 tumors treated with PEDF-PLGANPs (P-P) while none was found in CT26 tumors from $\mathrm{AAV}_{2^{-}}$ PLGANPs (A-P) or NS treated mice.

PLGANPs group obviously increased compared with that of $\mathrm{AAV}_{2}$-PLGANPs or NS group (Fig. 5A). Apoptotic index was carried out to evaluate quantitative comparison of apoptotic cells. The rate of PEDF-PLGANPs group was significantly higher than that of the other two groups $(\mathrm{p}<0.05)$ (Fig. 5B). In addition, hematoxylin and eosin (H\&E) staining was performed. Obvious necrosis could be seen in CT26 tumors of the PEDF-PLGANP group while less necrotic cells were seen in the $\mathrm{AAV}_{2}$-PLGANPs or the NS group (Fig. 5C).

The paraffin sections of CT26 tumors were stained with CD31 antibody to determine whether the growth inhibition of CT26 tumors was also related to the anti-angiogenesis activity of PEDF gene in PLGANPs. A large number of CD31 immunoreactive microvessels were seen in the sections of $\mathrm{NS}$ or $\mathrm{AAV}_{2}$-PLGANP-treated CT26 tumors while slight immunoreactive microvessels were observed in the sections of PEDF-PLGANPs treated CT26 tumors (Fig. 5D). As shown in Fig. 5E, the MVD of PEDF-PLGANP-treated CT26 tumors exhibited a significant decrease compared with that of NS or $\mathrm{AAV}_{2}$-PLGANP-treated CT26 tumors $(\mathrm{p}<0.05)$.

\section{Discussion}

Non-viral vectors, which are gaining interest, have been reported to have beneficial effects in gene delivery in vitro and in vivo $(28,29)$. However, some non-viral vectors are limited in use in vivo due to high toxicity because of their non-biodegradation. To develop non-toxic non-viral gene delivery systems is one of the most important research goals in this field. The transfer of PEDF gene also needs a safe and effective delivery system. In the present study, we chose PEI and bPLGANPs to evaluate their cytotoxicity to CT26s and we found that bPLGANPs showed less cytotoxicity than PEI to CT26s (Fig. 1D), which was consistent with the lowtoxicity feature of PLGANPs reported previously (30). Therefore, PLGANPs were used to mediate PEDF gene to observe its influence on CT26s in vitro and in vivo in our study. In addition, the expression of PEDF protein in vitro was examined by SDS-PAGE in our study. PEDF protein could be probed in CT26s after PEDF-PLGANP treatment (Fig. 2A), which suggested that PLGANPs could transfer PEDF gene to the CT26s effectively and PEDF gene was successfully expressed.

In the present study, the growth of CT26 tumors exhibited an inhibitory effect on PEDF-PLGANP-treated mice (Fig. 3). 
A

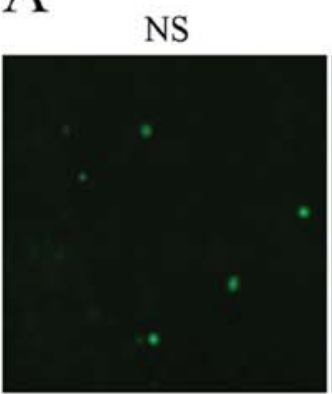

$\mathrm{AAV}_{2}-\mathrm{PLGANPS}$

PEDF-PLGANPs
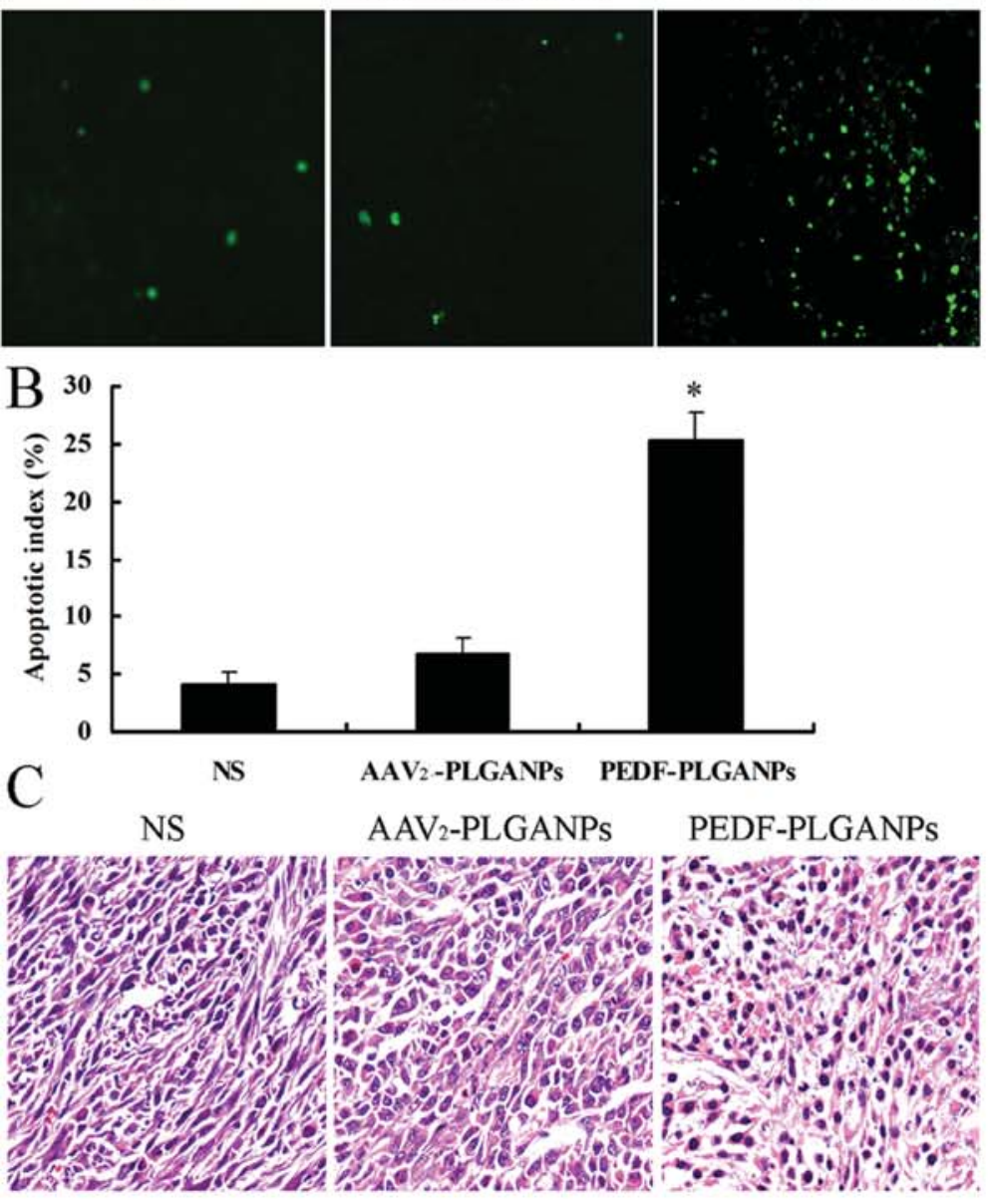

\section{$\mathrm{D} \quad \mathrm{NS} \quad \mathrm{AAV}_{2}$-PLGANPS PEDF-PLGANPS}

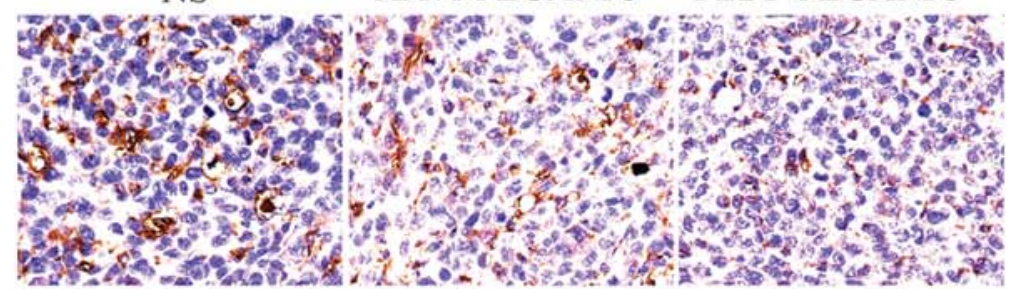

$\mathrm{E}$

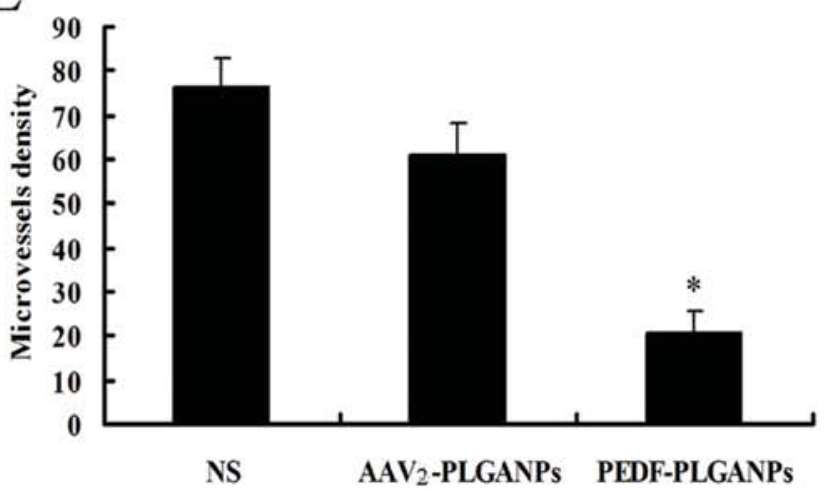

Figure 5. Related factors to the growth inhibition of CT26 tumors. (A) Apoptotic cells within CT26 tumors were detected by TUNEL assay. Apoptotic CT26s (green) of NS, $\mathrm{AAV}_{2}$-PLGANPs and PEDF-PLGANPs group were, respectively, observed under a fluorescence microscope (original magnification, $\mathrm{x} 200$ ). (B) The apoptotic indexes (\%) of CT26 tumors treated with NS, AAV ${ }_{2}$-PLGANPs and PEDF-PLGANPs. (C) CT26 tumors from NS, AAV $\mathrm{V}_{2}$-PLGANPs and PEDF-PLGANP-treated mice were stained with H\&E (original magnification, $x 400$ ). (D) CD31 immunoreactive microvessels were seen from the sections of NS, $\mathrm{AAV}_{2}$-PLGANPs and PEDF-PLGANP-treated tumors. (E) The quantitative data manifested that MVD per 400 field of PEDF-PLGANPtreated tumors had a significant decrease compared with that of $\mathrm{NS}$ or $\mathrm{AAV}_{2}-\mathrm{PLGANP}$-treated tumors. The data are expressed as mean $\pm \mathrm{SD}$. ${ }^{*} \mathrm{P}<0.05$ compared with $\mathrm{AAV}_{2}$-PLGANPs or NS group. 
We confirmed that PEDF gene loaded in PLGANPs could be successfully expressed in vivo by SDS-PAGE (Fig. 4). PEDF is a multifunctional protein with therapeutic effect on tumors. It can repress the growth of tumors directly. When PEDF gene interacts with tumor cells, expressed PEDF protein has the ability to induce tumor cell apoptosis directly (31). In our study, PEDF-PLGANPs were exposed to CT26s directly in vitro and in vivo. As a result, the CT26s exposed to PEDFPLGANPs had a noticeable apoptotic peak compared with the other two groups (Fig. 2B) in vitro. Furthermore, PEDFPLGANPs were applied to treat the primary CT26 tumors in vivo. $\mathrm{AAV}_{2}$-PLGANPs and NS were the control groups. When the mice were sacrificed, tumor tissue paraffin sections were submitted to TUNEL assay. The results exhibited that the number of apoptotic CT26s from the PEDF-PLGANPtreated mice was obviously higher than that of $\mathrm{AAV}_{2^{-}}$ PLGANPs or NS treated group (Fig. 5A and B), which was consistent with the finding in vitro. One way of direct inhibition of PEDF gene to tumors is via anti-proliferative effect which finally achieves apoptosis. The activity is likely to be related to a distinct functional epitope sequence of PEDF gene, 34-mer peptide sequence (32). In our study, the apoptosis of CT26s in vitro and in vivo possibly correlates to 34-mer peptide sequence of PEDF gene and the question should be further studied.

Indirectly, PEDF gene has a profound effect on tumor neovascularization to inhibit the growth of tumors $(4,6)$. Neovascularization is particularly critical to the growth of solid malignant tumors (33), including colon carcinoma, because successful growth of tumors requires the establishment of sufficient nutrition and oxygen by vascular supply (34). PEDF protein is one of endogenous anti-angiogenic agents that are extremely important for inhibiting neovascularization. Although the molecular mechanism by which PEDF protein educes its anti-angiogenic activity is yet largely unknown, it has been demonstrated that PEDF protein can decrease the microvascularity and blood supply of tumor and thus cause tumor reduction. It has been reported that PEDF is involved in the control of melanoma and plays a great role in the inhibition of tumor angiogenesis (35). Besides, PEDF was able to significantly inhibit the growth of glioma cells in vitro and in vivo (36); the decrease of PEDF could promote tumor growth and metastasis (37). In our study, CD31 staining targeted at new vessels was used to identify whether the CT26 tumor growth inhibition was also associated with the decrease of tumor neovascularization. As a result, the sections from PEDF-PLGANP-treated mice showed least number of new vessels in the three groups (Fig. 5D and E). In vitro, PEDF-PLGANPs were exposed to HUVECs, which showed significant proliferation inhibition while other two control groups had no significantly changes (Fig. 2C). The inhibition of HUVEC proliferation was dose-dependent. The results exhibited that the growth inhibition of CT26 tumors was also associated with the decrease of new vessels and angiogenesis inhibition induced by PEDF-PLGANPs, possibly via the death of vascular endothelial cells.

However, some questions remain. PEDF-PLGANPs were mainly used for the therapy of CT26 primary tumors, its effect on late stage tumor growth and metastasis tumor are still unknown; it is not clear whether PEDF-PLGANPs could induce immune response that may be associated with the growth inhibition of tumors.

In summary, our study found, for the first time, that PEDF gene loaded in PLGANPs could effectively inhibit the growth of CT26 tumors. The inhibitory effects of PEDFPLGANPs on CT26s were associated with apoptosis of CT26s, the decrease of MVD and the inhibition of angiogenesis which were necessary for the growth of tumors. The use of the PEDF gene and PLGANPs were a safe and effective gene delivery system for therapy of colon carcinoma. It may be an innovative approach for future therapeutic strategies also against other tumors.

\section{Acknowledgements}

The study is supported by Program for New Century Excellent Talents in University (NCET), the National Key Basic Research Project of China (973) (2010CB529900), the National Natural Science Foundation of China (No. 30901868 and No. 30772668) and National 863 Project (No. 2007AA021810). We thank Dr Xiancheng Chen (Department of Gynecology and Obstetrics, Second West China Hospital, China) for the immunochemistry technical support.

\section{References}

1. Anderson WF: Human gene therapy. Nature 392: 25-30, 1998.

2. Zhang L, Chen J, Ke Y, Mansel RE and Jiang WG: Downregulation of PEDF expression by ribozyme transgene in endothelial and lung cancer cells and its impact on angiogenesis in vitro. Oncol Rep 14: 1615-1619, 2005.

3. Dawson DW: Pigment epithelium-derived factor: a potent inhibitor of angiogenesis. Science 285: 245-248, 1999.

4. Yang LP, Cheng P, Peng XC, et al: Anti-tumor effect of adenovirus-mediated gene transfer of pigment epithelium-derived factor on mouse B16-F10 melanoma. J Exp Clin Cancer Res 28: 75, 2009.

5. Abe R, Shimizu T, Yamagishi S, et al: Overexpression of pigment epithelium-derived factor decreases angiogenesis and inhibits the growth of human malignant melanoma cells in vivo. Am J Pathol 164: 1225-1232, 2004.

6. Doll JA, Stellmach VM, Bouck NP, et al: Pigment epitheliumderived factor regulates the vasculature and mass of the prostate and pancreas. Nat Med 9: 774-780, 2003.

7. Kojiro M, Hiroki I, Daisuke N, Keisuke H, Kazuhiko N and Katsumi E: Antiangiogenic property of pigment epitheliumderived factor in hepatocellular carcinoma. Hepatology 40: 252-259, 2004.

8. Garcia M, Fernandez Garcia NI, Rivas V, et al: Inhibition of xenografted human melanoma growth and prevention of metastasis development by dual antiangiogenic/antitumor activities of pigment epithelium-derived factor. Cancer Res 64: 5632-5642, 2004.

9. Hase R, Miyamoto M, Uehara H, et al: Pigment epitheliumderived factor gene therapy inhibits human pancreatic cancer in mice. Clin Cancer Res 11: 8737-8744, 2005.

10. Guan M, Yam HF, Su B, et al: Loss of pigment epithelium derived factor expression in glioma progression. J Clin Pathol 56: 277-282, 2003.

11. Guan M, Pang CP, Yam HF, et al: Inhibition of glioma invasion by overexpression of pigment epithelium-derived factor. Cancer Gene Ther 11: 325-332, 2004.

12. Christiano R: Viral and non-viral vectors for cancer gene therapy. Anticancer Res 18: 3142-3246, 1998.

13. Abdallah B, Hassan A, Benoist C, Goula D, Behr JP and Demeneix BA: A powerful non-viral vector for in vivo gene transfer into the adult mammalian brain: polyethylenimine. Hum Gene Ther 7: 1947-1954, 1996.

14. Benns JM, Maheshwari A, Furgeson DY, Mahato RI and Kim SW: Folate-PEG-folate-graft-polyethylenimine-based gene delivery. J Drug Target 9: 123-139, 2001. 
15. Fischer D, Bieber T, Li Y, Elsasser HP and Kissel T: A novel non-viral vector for DNA delivery based on low molecular weight, branched polyethylenimine: effect of molecular weight on transfection efficiency and cytotoxicity. Pharm Res 16: 1273-1279, 1999.

16. Gosselin MA, Guo W and Lee RJ: Efficient gene transfer using reversibly crosslinked low molecular weight polyethylenimine. Bioconjug Chem 12: 989-994, 2001.

17. Song XR, Cai Z, Zheng Y, et al: Reversion of multidrug resistance by co-encapsulation of vincristine and verapamil in PLGANPs. Eur J Pharm Sci 37: 300-305, 2009.

18. Hedley ML, Curley J and Urban R: Microspheres containing plasmid-encoded antigens elicit cytotoxic T-cell responses. Nat Med 4: 365-368, 1998

19. Perez C, Sanchez A, Putnam D, Ting D, Langer R and Alonso MJ Poly (lactic acid)- poly (ethylene glycol) nanoparticles as new carriers for the delivery of plasmid DNA. J Control Release 75: 211-224, 2001

20. Panyam J, Zhou WZ, Prabha S, Sahoo SK and Labhasetwar V: Rapid endolysosomal escape of poly (d,l-lactide-co-glycolide) nanoparticles: implications for drug and gene delivery. FASEB J 16: 1217-1226, 2002.

21. Freytag T, Dashevsky A, Tillman L, Hardee GE and Bodmeier R: Improvement of the encapsulation efficiency of oligonucleotidecontaining biodegradable microspheres. J Control Release 69: 197-207, 2000.

22. Song XR, Zhao Y, Hou SX, et al: Dual agents loaded PLGANPs: systematic study of particle size and drug entrapment efficiency. Eur J Pharm Biopharm 69: 445-453, 2008.

23. Cohen H, Levy RJ, Gao J, Fishbein I, Kousaev V and Sosnowski S: Sustained delivery and expression of DNA encapsulated in polymeric nanoparticles. Gene Ther 7: 1896-1905, 2000.

24. Li G, Tian L, Hou JM, et al: Improved therapeutic effectiveness by combining recombinant $\mathrm{CXC}$ chemokine ligand 10 with Cisplatin in solid tumors. Clin Cancer Res 11: 4217-4224, 2005.

25. Wang L, Schmitz V, Perez-Mediavilla A, Izal I, Prieto J and Qian C: Suppression of angiogenesis and tumor growth by aden-viral-mediated gene transfer of pigment epitheliumderived factor. Mol Ther 8: 72-79, 2003

26. Peng XC, Yang L, Yang LP, et al: Efficient inhibition of murine breast cancer growth and metastasis by gene transferred mouse survivin Thr34 $\rightarrow$ Ala mutant. J Exp Clin Cancer Res 27: 46, 2008 .
27. Weidner N, Semple JP, Welch WR and Folkman J: Tumor angiogenesis and metastasis: correlation in invasive breast carcinoma. N Engl J Med 324: 1-8, 1991.

28. Lee M, Rentz J, Bikram M, Han S, Bull DA and Kim SW: Hypoxiainducible VEGF gene delivery to ischemic myocardium using watersoluble lipopolymer. Gene Ther 10: 1535-1542, 2003.

29. Abdallah B, Hassan A, Benoist C, Goula D, Behr JP and Demeneix BA: A powerful non-viral vector for in vivo gene transfer into the adult mammalian brain: polyethylenimine. Hum Gene Ther 7: 1947-1954, 1996.

30. Kang SW, Lim HW, Seo SW, Jeon O, Lee M and Kim BS: Nanosphere-mediated delivery of vascular endothelial growth factor gene for therapeutic angiogenesis in mouse ischemic limbs. Biomaterials 29: 1109-1117, 2008.

31. Takenaka K, Yamagishi S, Jinnouchi Y, Nakamura K, Matsui T and Imaizumi T: Pigment epithelium-derived factor (PEDF)induced apoptosis and inhibition of vascular endothelial growth factor (VEGF) expression in MG63 human osteosarcoma cells. Life Sci 77: 3231-3241, 2005.

32. Filleur S, Volz K, Nelius T, et al: Two functional epitopes of pigment epithelial-derived factor block angiogenesis and induce differentiation in prostate cancer. Cancer Res 65: 5144-5152, 2005.

33. Gimbrone MA Jr, Leapman SB, Cotran RS and Folkman J: Tumor dormancy in vivo by prevention of neovascularization. J Exp Med 136: 261-276, 1972.

34. Folkman J: Tumor angiogenesis: therapeutic implications. N Engl J Med 285: 1182-1186, 1971.

35. Abe R, Fujita Y, Yamagishi S and Shimizu H: Pigment epithelium-derived factor prevents melanoma growth via angiogenesis inhibition. Curr Pharm Des 14: 3802-3809, 2008.

36. Zhang T, Guan M, Xu C, Chen Y and Lu Y: Pigment epitheliumderived factor inhibits glioma cell growth in vitro and in vivo. Life Sci 81: 1256-1263, 2007.

37. Uehara H, Miyamoto M, Kato K, et al: Expression of pigment epithelium-derived factor decreases liver metastasis and correlates with favorable prognosis for patients with ductal pancreatic carcinoma. Cancer Res 64: 3533-3537, 2004. 